\title{
Introduction to Social Network Analysis
}

\author{
Panayiotis Zaphiris ${ }^{1}$ and Chee Siang $\mathrm{Ang}^{2}$ \\ ${ }^{1}$ Department of Multimedia \& Graphic Arts, Cyprus University of Technology, \\ Lemessos, 3603, Cyprus \\ ${ }^{2}$ Centre for HCI Design, City University London, EC1V 0HB, UK \\ pzaphiri@gmail.com, jimmybbq@gmail.com
}

\begin{abstract}
Social Network analysis focuses on patterns of relations between and among people, organizations, states, etc. It aims to describe networks of relations as fully as possible, identify prominent patterns in such networks, trace the flow of information through them, and discover what effects these relations and networks have on people and organizations. Social network analysis offers a very promising potential for analyzing human-human interactions in online communities (discussion boards, newsgroups, virtual organizations). This Tutorial provides an overview of this analytic technique and demonstrates how it can be used in Human Computer Interaction (HCI) research and practice, focusing especially on Computer Mediated Communication (CMC). This topic acquires particular importance these days, with the increasing popularity of social networking websites (e.g., youtube, myspace, MMORPGs etc.) and the research interest in studying them.
\end{abstract}

Keywords: Social Network Analysis, Social Computing, Computer Mediated Communication.

\section{Objectives}

This tutorial provides an overview of Social Network Analysis (SNA) and demonstrates through theory and practical case studies how it can be used in HCI (especially computer-mediated communication and $\mathrm{CSCW}$ ) research and practice. This topic becomes even more important these days with the increasing popularity of social networking websites (e.g. YouTube, Facebook, MySpace etc.) and social computing. As people increasingly use online communities for social interaction, new methods are needed to study these phenomena. SNA is a valuable contribution to HCI research as it gives an opportunity to study the complex patterns of online communication.

Social network theory views a network as a group of actors who are connected by a set of relationships. Actors are often people, but can also be nations, organizations, objects etc. Social Network Analysis (SNA) focuses on patterns of relations between these actors. It seeks to describe networks of relations as fully as possible. This includes teasing out the prominent patterns in such networks, tracing the flow of information through them, and discovering what effects these relations and networks have on people and organizations. It can therefore be used to study network patterns of 
organizations, ideas, and people that are connected via various means in an online environment.

Upon completion of this tutorial, attendees will:

1. Be able to understand the basics of social network analysis, its terminology and background (part 1)

2. Be able to transform communication data to network data (part 1)

3. Know practically how social network analysis (SNA) can be applied to HCI (especially CMC) analysis (part 2)

4. Get familiar with the use of standard SNA tools and software (part 2)

5. Be able to derive practical and useful information through SNA analysis that would help design an innovative and successful online community. (part 2)

\section{Content and Benefits}

The content of the tutorial is divided into two parts, each of which is structured in small groups to maximize the interaction among participants:

1. Part 1: Introduction to Social Network Analysis. Benefits: you will be exposed to the introduction of SNA, get familiar with the terminology and definitions of SNA.

2. Part 2: Practical uses of social network analysis (SNA). Benefits: Through a series of interactive exercises, a number of case studies will be demonstrated and discussed. Case studies will draw from diverse areas (e.g. use of SNA to study age differences in CMC, use of SNA in universal design and research). Ways of using SNA to study new forms of CMC such as MMORPGs, Wikis, blogs etc. will also be discussed.

\section{Presentation Format}

We will deliver the tutorial content in various presentation formats. The presentation of lecture material will include several different media. The presentation will be accompanied by videos and images to demonstrate examples. Furthermore, we will present popular SNA tools in order to provide the audience with hands on experience of using SNA software. Especially the more practical parts of the tutorial will be held in an interactive atmosphere. Discussions and exercises will give attendees the opportunity to bring in knowledge and questions from their own research and work area. As SNA can be applied in a variety of different research and work areas, we will allow for time to discuss and elaborate on the possibilities of SNA to the research or working areas of the participants.

\section{Target Audience}

We welcome practitioners and academics interested in computer mediated communication, universal design, especially researchers and practitioners who are interested in domains that social network analysis can be applied. 